\title{
INVENTÁRIO DA ENTOMOFAUNA DE ECOSSISTEMAS DA ÁREA DE PROTEÇÃO AMBIENTAL DO ARARIPE COM BANDEJAS D'ÁGUA AMARELAS
}

\author{
F. R. AZEVEDO ${ }^{1 *}$, E. S. MOURA ${ }^{2}$, R. AZEVEDO ${ }^{1}$, C. M. SANTOS ${ }^{2}$ e D. R. NERE $^{1}$ \\ ${ }^{1}$ Universidade Federal do Ceará - UFC \\ ${ }^{2}$ Universidade Federal de Viçosa - UFV \\ razevedo@ufc.br*
}

Artigo submetido em julho/2014 e aceito em junho/2015

DOI: 10.15628/holos.2015.2249

\section{RESUMO}

Com o objetivo de realizar um inventário da entomofauna dos ecossistemas da Área de Proteção Ambiental da Chapada do Araripe, localizada na região do Cariri cearense, (Arajara Park, Área Agrícola, Mata Úmida e Fragmento de Floresta), foi realizado um estudo utilizando bandejas d'água de cor amarela, visando a caracterização das famílias capturadas, no período de 03 de setembro de 2010 a fevereiro de 2011 (estação seca) e 22 de março de 2011 a agosto de 2011 (estação chuvosa). A estação seca da Área de Proteção Ambiental da Chapada do Araripe favorece a maior ocorrência de famílias abundantes que a estação chuvosa devido à alteração da disponibilidade de condições e recursos, propiciando a coexistência de espécies generalistas. A ordem Coleoptera é a mais rica em famílias nas duas estações do ano tendo como papel ecológico os Crysomelidae como fitófagas e Nitidulidae como decompositoras de frutas. Os Diptera são numerosos na estação chuvosa atuando no sistema ecológico as moscas frugívoras (Drosophilidae), decompositoras de carcaças de animais (Muscidae) e predadoras (Dolychopodidae), enquanto que os Tipulidae e Chloropidae predominam na estação seca da mata úmida. Os Hemiptera da família Cicadellidae (cigarrinhas) ocorrem abundantemente na área agrícola e no fragmento de floresta por serem ecossistemas antropizados para a implantação de culturas agrícolas e pastagens. Na estação seca os Hymenoptera da família Formicidae (formigas) predominam e ocorrem em maior abundância em todos os ecossistemas por serem cosmopolitas e bem adaptados, além de apresentarem hábitos alimentares generalistas. A bandeja d'água amarela foi eficiente na captura de insetos edáficos, demonstrando nas avaliações das coletas uma considerável diversidade de famílias em todos os ecossistemas avaliados assim como nas estações seca e chuvosa, permitindo conhecer a entomofauna da Área de Proteção Ambiental do Araripe e fornecendo uma base de informações consolidada para estudos futuros.

PALAVRAS-CHAVE: Bioindicadores, Armadilhas d"agua, Levantamentos, APA-Araripe.

\section{INVENTORY OF INSECT FAUNA OF ECOSYSTEM FROM ENVIRONMENTAL PROTECTION AREA OF ARARIPE WITH WATER TRAPS YELLOW}

\begin{abstract}
With the goal of realize one survey of insect fauna of ecosystems from Environmental Protection Area of Araripe, located in Cariricearense (Arajara Park, Farm Area, Humid Forest and Forest Patch), one study was been realized using yellow traps of water aiming characterize the families captured, during September 3TH 2010 to February 2011 (dry season) and from March 22TH to August 2011 (rainy season). The dry season of the Environmental Protection Area of Araripe contributes to a bigger occurrence of abundant families than rainy season due to changes in conditions and resources availability, allowing the coexistence of generalist species. The Coleoptera order is the most rich in number of families on the two seasonal seasons, which Crysomelidae and Nitidulidade exhibiting an ecological role as phytophagous and fruit decomposer, respectively. The Diptera are abundant on rainy season, acting in ecological system and
\end{abstract}

been represented by frugivorus flies (Drosophilidae), animal carcasses decomposer (Muscidae) and predators (Dolychopodidade), while the Tipulidae and the Chloropidae were been predominant on dry season of the humid forest. The leafhoppers (Hemiptera, Cicadellidae) are abundant in the farm area and in the forest patch due these ecosystems are been anthropogenic for areas of agricultural cultivation and pastures. On the dry season, the ants (Hymenoptera, Formicidae) predominate and occurs with great abundance in all ecosystems due been cosmopolitan and well adapted, also present generalist feed habit. The yellow tray water was efficient on edaphic insects capture, exhibiting a meangninful family diversity in all ecosystems evaluated on dry and rainy season, allowing know the entomofauna's of Área de Proteção Ambiental do Araripe and allowing a database to future studies.

KEYWORDS: Bioindicators, watertraps, Surveys, APA-Araripe. 


\section{INTRODUÇÃO}

A importância dos insetos nos ecossistemas terrestres se justifica pelo seu envolvimento na decomposição de matéria orgânica, na ciclagem de nutrientes, no fluxo de energia, na polinização e na dispersão de sementes, além de serem reguladores de populações de plantas, de animais e de outros organismos (LOPES, 2008).

A dinâmica populacional dos insetos é altamente influenciada pela heterogeneidade dentro de um mesmo habitat (THOMAZINI; THOMAZINI, 2000). Estes dados auxiliam na conservação da biodiversidade, pois é um grupo que ocorre em praticamente todos os ambientes graças as suas peculiaridades estruturais e fisiológicas que permitem adaptações a condições ambientais bastante distintas (LOPES, 2008).

Assim, a entomofauna de uma região depende do habitat em conjunto das condições e recursos oferecidos por este (BEGON et al., 2008). A expansão das áreas urbanas sobre ambientes naturais ocasiona a destruição de inúmeros microhabitats de diversas espécies. Geralmente a fauna urbana é pouco diversificada e apresenta algumas poucas espécies dominantes. Essas podem atingir uma biomassa muitas vezes superior à observada em ambientes naturais, por encontrar na cidade um novo ambiente a ser explorado (ISERHARD et al., 2001).

É importante reconhecer a entomofauna de certas áreas para que haja um constante acompanhamento dos impactos da ação antrópica nessas comunidades, já que o número de ordens, famílias e espécies de insetos diminui com a elevação do nível de antropização do ambiente (THOMANZINI; THOMANZINI, 2002).

Diante desse contexto, a utilização de insetos em inventários e estudos ambientais, tornase essencial para o monitoramento da diversidade de espécies, onde se consegue averiguar as alterações ocasionadas pelo próprio ambiente, bem como, as causadas por antropização, que ameaçam a integridade dos ecossistemas (NASCIMENTO et al., 2001). Os inventários e o monitoramento dos insetos nos ecossistemas permitem as prevenções ou remediações de impactos nos diferentes ambientes. Assim, à medida que ocorrem o resgate da diversidade vegetal e o equilíbrio ambiental, também os insetos respondem em diversidade e densidade, cumprindo a sua função indicadora (WINK et al., 2005).

O uso de armadilhas é mais fácil e menos oneroso para levantamento de insetos (MELO et al., 2001), sendo que, a bandeja d'água de cor amarela é usada como uma armadilha atrativa que coleta insetos atraídos pela cor e que pousam no meio líquido. Araújo (2009) também as utilizou para monitorar as densidades populacionais de Aphis gossypii Glover no algodoeiro e Tognon et al., (2009) para a identificação de espécies de cigarrinhas como potenciais vetores de Xylella fastidiosa no Rio Grande do Sul.

Diante do exposto, o objetivo deste trabalho foi realizar um inventário da entomofauna dos ecossistemas da Área de Proteção Ambiental da Chapada do Araripe.

\section{MATERIAL E MÉTODOS}




\section{1 Áreas de estudo}

O inventário foi realizado na Área de Proteção Ambiental da Chapada do Araripe, (APA), em Barbalha-CE, localizada na região do Cariri cearense, no período de 03 de setembro de 2010 a fevereiro de 2011 (estação seca) e 22 de março de 2011 a agosto de 2011 (estação chuvosa), em quatro ecossistemas distintos: Mata Úmida (71'56" S e 3922'12,7" W), Fragmento de Floresta (7010'47" S e 3923'00" W),área turística (Arajara Park) (719'59,1" S e 3924'39,3" W) e uma Área Agrícola (719'50,7”' S e 3924'40,9” W).

\subsection{Instalação das armadilhas e coleta dos insetos}

Foram instaladas quatro bandejas dispostas em quadrado equidistantes $25 \mathrm{~cm}$ uma da outra $\left(625 \mathrm{~m}^{2}\right)$ por ecossistema. Estas foram colocadas no solo, contendo no seu interior $500 \mathrm{~mL}$ de uma solução de formol a $5 \%$ e algumas gotas de detergente neutro para quebrar a tensão superficial da água. Nas bordas das armadilhas foram feitos orifícios onde foram colocadas telas finas para que, em caso de chuva, o excesso de água na bandeja não levasse os insetos coletados a transbordarem, perdendo-se.

As coletas foram realizadas semanalmente com o auxílio de peneiras plásticas e pinceis finos e os insetos foram levados ao Laboratório de Entomologia Agrícola da Universidade Federal do Cariri para serem identificados em nível de ordem e família, utilizando-se chaves ilustradas de identificação (BUZZI, 2008; TRIPLEHORN; JONNSON, 2011).

\section{RESULTADOS E DISCUSSÃO}

\subsection{Composição da entomofauna}

Foram coletados insetos pertencentes a nove ordens, distribuídos em 69 famílias. A ordem Coleoptera apresentou a maior riqueza, com 18 famílias coletadas, representando $26 \%$ do total de famílias coletadas e esta foram seguidas pela ordem Diptera com 15 famílias, representando $22 \%$ do total, pela ordem Hemiptera com 14 famílias e $20 \%$ do total e Hymenoptera com 12 famílias, representando $17 \%$ do total. As ordens menos representativas foram Orthoptera com seis famílias e $9 \%$ do número total e Blattodea, Neuroptera, Isoptera e Odonata com apenas uma família coletada referente a $1 \%$ do total cada uma (Tabela 1 ).

Tabela 1: Abundância dos indivíduos coletados nas estações seca e chuvosa dos quatro ecossistemas da Área de Proteção Ambiental da Chapada do Araripe. Barbalha-CE, 2010/2011.

\begin{tabular}{|c|c|c|c|c|c|c|c|c|c|c|c|c|c|c|}
\hline \multirow[b]{2}{*}{ Ordens } & \multirow[b]{2}{*}{ Famílias } & \multicolumn{3}{|c|}{ Arajara Park } & \multicolumn{3}{|c|}{ Área Agrícola } & \multicolumn{3}{|c|}{ Mata Úmida } & \multicolumn{3}{|c|}{ Fragmento } & \multirow[b]{2}{*}{$\begin{array}{l}\text { Total } \\
\text { geral }\end{array}$} \\
\hline & & $\mathrm{S}$ & $\mathrm{C}$ & Total & $S$ & C & Total & $S$ & C & Total & $S$ & C & Total & \\
\hline \multirow{7}{*}{ Coleoptera } & Bostrychidae & 1 & 9 & 10 & 1 & 54 & 55 & 4 & 64 & 68 & 0 & 46 & 46 & 179 \\
\hline & Bruchidae & 0 & 0 & 0 & 0 & 0 & 0 & 0 & 0 & 0 & 1 & 1 & 2 & 2 \\
\hline & Buprestidae & 0 & 0 & 0 & 1 & 0 & 1 & 2 & 0 & 2 & 0 & 0 & 0 & 3 \\
\hline & Carabidae & 8 & 17 & 25 & 12 & 2 & 14 & 9 & 2 & 11 & 1 & 2 & 3 & 53 \\
\hline & Cerambycidae & 0 & 0 & 0 & 2 & 1 & 3 & 0 & 3 & 3 & 0 & 2 & 2 & 8 \\
\hline & Chrysomelidae & 16 & 125 & 141 & 35 & 18 & 53 & 10 & 25 & 35 & 32 & 24 & 56 & 285 \\
\hline & Coccinellidae & 0 & 0 & 0 & 2 & 0 & 2 & 1 & 0 & 1 & 20 & 16 & 36 & 39 \\
\hline
\end{tabular}




\begin{tabular}{|c|c|c|c|c|c|c|c|c|c|c|c|c|c|c|}
\hline \multirow[b]{2}{*}{ Ordens } & \multirow[b]{2}{*}{ Famílias } & \multicolumn{3}{|c|}{ Arajara Park } & \multicolumn{3}{|c|}{ Área Agrícola } & \multicolumn{3}{|c|}{ Mata Úmida } & \multicolumn{3}{|c|}{ Fragmento } & \multirow[b]{2}{*}{$\begin{array}{l}\text { Total } \\
\text { geral }\end{array}$} \\
\hline & & $\mathrm{S}$ & C & Total & $S$ & C & Total & $\mathrm{S}$ & C & Total & $S$ & C & Total & \\
\hline & Curculionidae & 7 & 3 & 10 & 10 & 8 & 18 & 41 & 45 & 86 & 0 & 0 & 0 & 114 \\
\hline & Elateridae & 3 & 7 & 10 & 2 & 1 & 3 & 7 & 3 & 10 & 1 & 1 & 2 & 25 \\
\hline & Hydrophilidae & 0 & 0 & 0 & 0 & 1 & 1 & 1 & 0 & 1 & 0 & 0 & 0 & 2 \\
\hline & Lagriidae & 0 & 0 & 0 & 0 & 2 & 2 & 0 & 0 & 0 & 0 & 0 & 0 & 2 \\
\hline & Lampyridae & 1 & 0 & 1 & 0 & 0 & 0 & 0 & 0 & 0 & 0 & 0 & 0 & 1 \\
\hline & Nitidulidae & 35 & 24 & 59 & 60 & 8 & 68 & 146 & 74 & 220 & 15 & 18 & 33 & 380 \\
\hline & Scarabaeidae & 3 & 2 & 5 & 2 & 0 & 2 & 2 & 0 & 2 & 22 & 3 & 25 & 34 \\
\hline & Scolytidae & 50 & 18 & 68 & 0 & 68 & 68 & 12 & 13 & 25 & 36 & 0 & 36 & 197 \\
\hline & Silphidae & 0 & 0 & 0 & 2 & 0 & 2 & 2 & 0 & 2 & 1 & 0 & 1 & 5 \\
\hline & Staphylinidae & 2 & 2 & 4 & 2 & 4 & 6 & 5 & 0 & 5 & 0 & 1 & 1 & 16 \\
\hline & Tenebrionidae & 0 & 1 & 1 & 0 & 0 & 0 & 1 & 0 & 1 & 0 & 0 & 0 & 2 \\
\hline \multirow{15}{*}{ Diptera } & Asilidae & 2 & 0 & 2 & 2 & 5 & 7 & 0 & 0 & 0 & 12 & 4 & 16 & 25 \\
\hline & Bombyliidae & 0 & 0 & 0 & 2 & 0 & 2 & 0 & 0 & 0 & 0 & 0 & 0 & 2 \\
\hline & Calliphoridae & 3 & 0 & 3 & 1 & 1 & 2 & 9 & 0 & 9 & 0 & 0 & 0 & 14 \\
\hline & Chloropidae & 100 & 36 & 136 & 80 & 262 & 342 & 302 & 58 & 360 & 85 & 43 & 128 & 966 \\
\hline & Culicidae & 25 & 10 & 35 & 29 & 39 & 68 & 8 & 9 & 17 & 5 & 0 & 5 & 125 \\
\hline & Dolichopodidae & 33 & 24 & 57 & 47 & 140 & 187 & 1 & 2 & 3 & 0 & 0 & 0 & 247 \\
\hline & Drosophilidae & 59 & 6 & 65 & 316 & 121 & 437 & 151 & 2 & 153 & 0 & 0 & 0 & 655 \\
\hline & Micropezidae & 1 & 1 & 2 & 28 & 15 & 43 & 5 & 0 & 5 & 0 & 0 & 0 & 50 \\
\hline & Muscidae & 0 & 1 & 1 & 213 & 14 & 227 & 378 & 8 & 386 & 29 & 3 & 32 & 646 \\
\hline & Otitidae & 43 & 2 & 45 & 5 & 0 & 5 & 1 & 0 & 1 & 2 & 0 & 2 & 53 \\
\hline & Sarcophagidae & 42 & 12 & 54 & 51 & 48 & 99 & 87 & 16 & 103 & 128 & 5 & 133 & 389 \\
\hline & Stratiomydae & 2 & 5 & 7 & 15 & 11 & 26 & 2 & 11 & 13 & 19 & 5 & 24 & 70 \\
\hline & Tabanidae & 2 & 0 & 2 & 2 & 16 & 18 & 0 & 0 & 0 & 2 & 0 & 2 & 22 \\
\hline & Tephritidae & 5 & 5 & 10 & 3 & 6 & 9 & 0 & 1 & 1 & 12 & 3 & 15 & 35 \\
\hline & Tipulidae & 121 & 4 & 125 & 1 & 3 & 4 & 4.389 & 1 & 4.390 & 5 & 5 & 10 & 4.529 \\
\hline \multirow{14}{*}{ Hemiptera } & Aphididae & 0 & 0 & 0 & 10 & 0 & 10 & 0 & 0 & 0 & 0 & 0 & 0 & 10 \\
\hline & Belostomatidae & 2 & 0 & 2 & 0 & 0 & 0 & 0 & 0 & 0 & 0 & 0 & 0 & 2 \\
\hline & Cercopidae & 0 & 4 & 4 & 0 & 0 & 0 & 0 & 4 & 4 & 0 & 0 & 0 & 8 \\
\hline & Cicadellidae & 119 & 59 & 178 & 390 & 155 & 545 & 225 & 160 & 385 & 903 & 234 & 1.137 & 2.245 \\
\hline & Cicadidae & 0 & 1 & 1 & 2 & 0 & 2 & 0 & 2 & 2 & 0 & 24 & 24 & 29 \\
\hline & Coreidae & 38 & 8 & 46 & 13 & 6 & 19 & 0 & 2 & 2 & 2 & 4 & 6 & 73 \\
\hline & Cydnidae & 9 & 0 & 9 & 0 & 0 & 0 & 0 & 0 & 0 & 0 & 1 & 1 & 10 \\
\hline & Fulgoridae & 0 & 0 & 0 & 0 & 0 & 0 & 0 & 0 & 0 & 0 & 1 & 1 & 1 \\
\hline & Membracidae & 0 & 0 & 0 & 1 & 0 & 1 & 0 & 0 & 0 & 1 & 1 & 2 & 3 \\
\hline & Miridae & 1 & 0 & 1 & 0 & 0 & 0 & 0 & 0 & 0 & 9 & 0 & 9 & 10 \\
\hline & Pentatomidae & 2 & 1 & 3 & 3 & 5 & 8 & 2 & 2 & 4 & 0 & 3 & 3 & 18 \\
\hline & Reduviidae & 1 & 2 & 3 & 1 & 4 & 5 & 1 & 0 & 1 & 0 & 1 & 1 & 10 \\
\hline & Rhophalidae & 1 & 3 & 4 & 3 & 0 & 3 & 1 & 0 & 1 & 3 & 0 & 3 & 11 \\
\hline & Scutelleridae & 1 & 0 & 1 & 0 & 0 & 0 & 0 & 0 & 0 & 0 & 1 & 1 & 2 \\
\hline Hymenoptera & Agaonidae & 0 & 0 & 0 & 0 & 0 & 0 & 1 & 0 & 1 & 0 & 0 & 0 & 1 \\
\hline
\end{tabular}




\begin{tabular}{|c|c|c|c|c|c|c|c|c|c|c|c|c|c|c|}
\hline \multirow[b]{2}{*}{ Ordens } & \multirow[b]{2}{*}{ Famílias } & \multicolumn{3}{|c|}{ Arajara Park } & \multicolumn{3}{|c|}{ Área Agrícola } & \multicolumn{3}{|c|}{ Mata Úmida } & \multicolumn{3}{|c|}{ Fragmento } & \multirow[b]{2}{*}{$\begin{array}{l}\text { Total } \\
\text { geral }\end{array}$} \\
\hline & & $\mathrm{S}$ & $\mathrm{C}$ & Total & $S$ & $\mathrm{C}$ & Total & $\mathrm{S}$ & $\mathrm{C}$ & Total & $S$ & $\mathrm{C}$ & Total & \\
\hline & Apidae & 0 & 2 & 2 & 13 & 2 & 15 & 1 & 0 & 1 & 3 & 6 & 9 & 27 \\
\hline & Braconidae & 0 & 0 & 0 & 11 & 0 & 11 & 0 & 0 & 0 & 0 & 0 & 0 & 11 \\
\hline & Eulophidae & 0 & 0 & 0 & 0 & 0 & 0 & 3 & 0 & 3 & 0 & 0 & 0 & 3 \\
\hline & Evaniidae & 0 & 2 & 2 & 1 & 2 & 3 & 11 & 0 & 11 & 2 & 1 & 3 & 19 \\
\hline & Formicidae & 313 & 156 & 469 & 381 & 168 & 549 & 838 & 136 & 974 & 908 & 11 & 919 & 2.911 \\
\hline & Hallictidae & 0 & 1 & 1 & 0 & 2 & 2 & 0 & 0 & 0 & 0 & 0 & 0 & 3 \\
\hline & Ichneumonidae & 8 & 24 & 32 & 73 & 150 & 223 & 247 & 95 & 342 & 155 & 70 & 225 & 822 \\
\hline & Mutillidae & 4 & 0 & 4 & 0 & 0 & 0 & 0 & 0 & 0 & 0 & 0 & 0 & 4 \\
\hline & Pompilidae & 0 & 0 & 0 & 0 & 0 & 0 & 0 & 0 & 0 & 1 & 0 & 1 & 1 \\
\hline & Pteromalidae & 16 & 3 & 19 & 45 & 0 & 45 & 24 & 10 & 34 & 5 & 0 & 5 & 103 \\
\hline & Vespidae & 23 & 10 & 33 & 71 & 65 & 136 & 71 & 13 & 84 & 121 & 9 & 130 & 383 \\
\hline \multirow{6}{*}{ Orthoptera } & Acrididae & 1 & 1 & 2 & 24 & 12 & 36 & 13 & 1 & 14 & 7 & 14 & 21 & 73 \\
\hline & Gryllacrididae & 0 & 0 & 0 & 1 & 1 & 2 & 0 & 0 & 0 & 1 & 1 & 2 & 4 \\
\hline & Gryllidae & 12 & 9 & 21 & 36 & 21 & 57 & 60 & 16 & 76 & 65 & 63 & 128 & 282 \\
\hline & Proscopiidae & 0 & 0 & 0 & 2 & 0 & 2 & 1 & 0 & 1 & 0 & 0 & 0 & 3 \\
\hline & Stenopelmatidae & 0 & 0 & 0 & 0 & 0 & 0 & 0 & 0 & 0 & 2 & 0 & 2 & 2 \\
\hline & Tettigoniidae & 1 & 1 & 2 & 2 & 3 & 5 & 0 & 0 & 0 & 0 & 0 & 0 & 7 \\
\hline Blattodea & Blattidae & 6 & 4 & 10 & 21 & 10 & 31 & 43 & 11 & 54 & 32 & 21 & 53 & 148 \\
\hline Isoptera & Termitidae & 6 & 0 & 6 & 19 & 0 & 19 & 25 & 0 & 25 & 6 & 0 & 6 & 56 \\
\hline Neuroptera & Chrysopidae & 0 & 1 & 1 & 1 & 6 & 7 & 1 & 0 & 1 & 0 & 0 & 0 & 9 \\
\hline Odonata & Libellulidae & 0 & 3 & 3 & 0 & 0 & 0 & 0 & 0 & 0 & 0 & 0 & 0 & 3 \\
\hline Total & & 1.128 & 609 & 1.737 & 2.050 & 1.460 & 3.510 & 7.144 & 789 & 7.933 & 2.654 & 648 & 3.302 & 16.482 \\
\hline
\end{tabular}

Azevedo et al., (2011), também observaram em seu trabalho com bandejas amarelas a predominância das ordens Coleoptera e Diptera com maiores representantes em total de famílias. Os besouros possuem grande importância ecológica, auxiliando na percepção das condições ambientais locais de uma fitofisionomia (CHUNG et al., 2000) e a sua diversidade está relacionada com a composição e a estrutura da vegetação, revelando um mecanismo natural de atração, abrigo e alimentação (SCHORN, 2000).

\subsection{Insetos coletados na estação seca}

Nessa estação, capturou-se um total de 12.976 insetos e a mata úmida foi responsável pelo maior número de indivíduos capturados com 7.144, representando 55,2\% do total (Tabela 1). Este ecossistema por se tratar de um ambiente equilibrado fornece melhores condições para o desenvolvimento e diversidade de insetos com pouca ou ausência da ação antrópica e também por apresentar uma vegetação densa, o que justifica a ocorrência de insetos de voo baixo (MOURA et al.,2012).

A família mais abundante nesse ambiente foi Tipulidae com 4.389 indivíduos. Isto pode ser explicado pelo fato desta família necessitar de habitats aquáticos ou semiaquáticos durante a fase larval, sendo estes, córregos, nascentes e bromélias entre outros. As larvas habitam o fundo 
dos rios e lagos, mesmo sendo abundantes, elas passam a maior parte do tempo escondidas no cascalho e nos detritos, colocando-as fora do alcance dos peixes. A riqueza desta família neste ambiente também pode ser justificada pela qualidade e quantidade dos recursos hídricos que este dispõe, não esquecendo também da preservação ambiental, pois é um ecossistema que faz parte da Floresta Nacional do Araripe (FLONA-Araripe) que é uma Unidade de Conservação Ambiental.

Em seguida, as formigas apresentaram 838 indivíduos. As cortadeiras (Myrmicinae, Attini), além de reduzirem de 4 a 17\% da produção anual de folhas de uma floresta como a Amazônica (WIRTH et al., 2003; ARAÚJO, 2004), criam clareiras de sub-bosque para a construção dos ninhos, aumentando a quantidade de luz que chega ao chão da floresta e modificando a composição de espécies e a estrutura das comunidades vegetais (FARJI-BRENER; ILLES 2000). As formigas são dominantes nos ecossistemas tanto pela riqueza de espécies quanto pelo número de indivíduos e são facilmente coletadas pelo hábito de forragear em grupo (SILVA et al., 2006). Em trabalho realizado com armadilhas de solo, Araújo et al., (2010) também observaram predominância das formigas nas armadilhas de coleta, assim como Guariento (2005) e Silva et al., (2006).

Os Diptera das famílias Muscidae, Chloropidae e Drosophilidae apresentaram uma abundância de 378, 302 e 151 insetos, respectivamente. Os adultos de Muscidae podem ser predadores, hematófagos, saprófagos ou necrófagos. As larvas ocupam habitats extremamente variados, tais como esterco de mamíferos e carne em putrefação, matéria orgânica vegetal e animal em decomposição, madeira, fungos, ninhos e tocas de mamíferos, entre outros, o que justifica sua ocorrência neste ecossistema, devido à presença de animais silvestres. As espécies com larvas saprófagas e coprófagas podem ser benéficas, contribuindo na reciclagem de desperdícios orgânicos com as folhas das árvores, enquanto as larvas carnívoras podem se alimentar de larvas de espécies sinantrópicas, desempenhando o papel de reguladores destas populações (CARVALHO et al., 1993). Os Chloropidae atingiram este número de abundância devido à existência de animais silvestres neste ecossistema. Podem causar miíases em anfíbios, como as larvas do gênero Batrachomyia, que se desenvolvem sob a pele de sapos e rãs (LEMCKERT, 2000). Os drosofilídeos são apontados como potenciais bioindicadores para a avaliação de impacto e monitoramento da recuperação florestal. Essa família é apenas uma das mais de 150 famílias que compõe a ordem Diptera (YEATES; WIEGMANN, 2005), mas encontra-se entre as mais diversas e amplamente distribuídas. Dentro dos ecossistemas esses insetos desempenham papel fundamental nas cadeias saprofíticas, uma vez que seus estádios imaturos alimentam-se de organismos fermentadores e na mata úmida existem diversas espécies de frutos silvestres.

Os Hymenoptera da família Ichneumonidae com 247 insetos coletados têm um papel ecológico importante, pois são parasitoides de estádios imaturos, principalmente larvas de outros insetos, o que explica sua alta abundância neste ecossistema, já que o mesmo dispõe de ambiente favorável e rico em alimentos para estes insetos.

São importantes no controle biológico, pois como parasitoides sempre matam o hospedeiro. Segundo Lasalle; Gauld (1993), eles ocupam vários níveis tróficos no seu desenvolvimento, sendo assim, mais sensíveis às mudanças ambientais. A modificação do ambiente acarreta em perda de habitat dos hospedeiros e consequente alteração na abundância destes inimigos naturais. 
Os Hemiptera da família Cicadellidae (cigarrinhas) com 225 insetos coletados, devido a seu hábito fitossuccívoro, representam um grupo com grande potencial para estudos relacionados à biodiversidade florestal e como indicadora das alterações na composição vegetal desse ambiente (COELHO, 2003). Nessa mesma vegetação e estação do ano, Azevedo et al. (2011) não coletaram nenhuma espécime dessa família na Floresta Nacional do Araripe.

Já os Coleoptera da família Nitidulidae tiveram uma abundância de 146 insetos e abriga pequenos besouros que medem entre 1,5 e 5,0 $\mathrm{mm}$ de comprimento, geralmente encontrados em substratos orgânicos como frutos em decomposição, carcaças de animais, flores e fungos (TRIPLEHORN; JONNSON, 2011), condições estas encontradas na mata úmida e, algumas de suas espécies apresentam hábito predador.

Seguido da mata úmida, observou-se um total de 2.654 indivíduos no Fragmento de Floresta, destacando-se em abundância as formigas e cigarrinhas com 908 e 903 insetos, respectivamente. Nesta estação, o fragmento de floresta consistiu no ecossistema que capturou um maior número de formigas. O processo de degradação, promovido pela expansão das áreas urbanas e fronteiras agrícolas causa a perda e a fragmentação das florestas tropicais, modificando a estrutura e a qualidade dos remanescentes florestais, importantes para a conservação da biodiversidade (ALENCAR et al., 2004). É sabido que a fragmentação das florestas pode levar a alterações do microclima e afetar direta ou indiretamente a dinâmica de populações e comunidades, podendo resultar em aumento ou redução da diversidade de espécies (THOMAZINI; THOMAZINI 2000).

As formigas são utilizadas como bioindicadores (SANTOS et al., 2006) e servem como modelo em estudos de biodiversidade devido à sua elevada dominância numérica e por serem facilmente amostradas (ALONSO; AGOSTI, 2000). Os formicídeos também são conhecidos por habitar desde o solo até as copas das árvores. No entanto, a maioria dos estudos se concentra na investigação de formigas que forrageiam e/ou nidificam na serapilheira (VEIGA-FERREIRA et al., 2005). Davidson et al. (2003) afirmaram que é importante estudar a mirmecofauna arborícola, porque em todas as regiões tropicais e subtropicais do mundo as formigas aparecem como o componente mais abundante deste habitat, havendo um grande potencial para a descoberta de novas espécies (LEWINSOHN et al., 2005). Outros estudos têm inovado ao descrever hábitos comportamentais de espécies arborícolas (DEL-CLARO et al., 2002), além da sua importância na manutenção da diversidade florestal.

Com relação à comparação entre o resultado de captura de diferentes armadilhas, testes realizados por Longino; Colwel (1997) indicaram que, para formigas, a termonebulização, Malaise e Berlese têm a mesma eficiência de captura. No entanto, termonebulização e Malaise têm uma baixa complementaridade, mas quando uma destas é utilizada juntamente com Berlese, há um ganho em diversidade, o que se equipara ao uso de bandejas d'água coloridas (TEIXEIRA, 2012).

No entanto, o desmatamento florestal causa a eliminação de muitas colônias de formigas e a diminuição das áreas de forrageamento no habitat, tornando os ambientes cada vez mais hostis para várias espécies de formigas (SCHOEREDER et al., 2004), enquanto outras podem ter sua abundância aumentada devido ao seu alto potencial de adaptação.

Seguido das formigas, os Hemiptera da família Cicadellidae apresentaram uma abundância de 903 insetos. Esses insetos preferem habitat constituído por vegetações baixas (VAZ et al., 2009) e, provavelmente, pelo fato da vegetação do fragmento apresentar plantas 
mais baixas, essa condição propiciou maior atividade de voo dessas cigarrinhas entre as plantas e maior captura nas bandejas, quando comparado com a da mata úmida.

Os Hymenoptera das famílias Ichneumonidae e Vespidae apresentaram abundâncias de 155 e 121 insetos, respectivamente. São inimigos naturais de muitos insetos e os vespídeos também contribuem para a polinização de muitas espécies de plantas, tanto potencialmente como efetivamente, sendo comprovadamente transportadores de pólen (GALLO et al., 2002). A utilização de várias técnicas ou a junção delas em um mesmo aparato coletor pode maximizar a coleta de espécies. Em um estudo em Minas Gerais, a utilização de frascos de coleta de cor amarela em Armadilhas Malaise tratadas com inseticida, juntamente com bandejas amarelas em sua porção inferior, foi responsável pela duplicação e até mesmo pela triplicação do número de himenópteros capturados (CAMPOS et al., 2000). Capturas com rede entomológica e armadilhas d'água se mostraram igualmente eficazes na captura de abelhas e vespas (MONSEVIÈIUS, 2004).

Os Diptera da família Sarcophagidae apresentaram 128 insetos e a sua presença neste ecossistema pode ser explicada pela presença de carcaças de animais como gado de corte e outros das quais estes insetos se alimentam e a ação humana nesta área de estudo devido ao desmatamento da floresta para implantação de pastagens e culturas.

A baixa diversidade de famílias abundantes em comparação com o ecossistema anterior se justifica pelo fato de a destruição das florestas tropicais e a sua fragmentação contribuírem para que muitas espécies de animais e vegetais desapareçam já que devido à perda e/ou modificação de seus habitats elas se tornam mais vulneráveis à extinção (KAGEYAMA; LEPSCHCUNHA, 2001).

$\mathrm{Na}$ área agrícola foram coletados 2.050 insetos e as cigarrinhas e formigas predominaram com 390 e 381 insetos, respectivamente. As formigas são abundantes nesse ecossistema que se encontra em desequilíbrio provocado pelo desmatamento para a implantação de culturas agrícolas, pois estes insetos são considerados pragas agrícolas neste ecossistema (NERE et al., 2011). Em trabalhos utilizando a armadilha batedor entomológico ("Beating sheets/ trays") em ecossistemas agrícolas de Portugal, Santos et al., (2006) também observaram predominância da família Formicidae, tanto em áreas orgânicas como áreas tratadas com defensivos químicos, na última o número de integrantes desta família foi menor do que em áreas orgânicas, o que aponta para a hipótese de estes indivíduos serem importantes representantes como bioindicadores da qualidade ambiental de um ecossistema (SANTOS, et al., 2006). Embora as formigas sejam consideradas um grupo-chave em ecossistemas agrícolas (PEREIRA et al., 2004), nomeadamente como bioindicadores de distúrbios, as informações relativas à efeitos da ação antrópica sobre este grupo é escassa (SANTOS, et al., 2006).

O uso do solo para diferentes fins, como na agricultura, pode influenciar suas características físicas, químicas e propriedades biológicas. No aspecto biológico, pode ocorrer uma seleção de grupos de artrópodes do solo, além de alterar a abundância e diversidade destes (LUZ et al., 2013), justificando assim a maior ocorrência da família Formicidae neste ecossistema. Alguns insetos, por exemplo, podem interagir com outros microrganismos, que se decompõem e mineralizam os detritos do solo (HÖFER et al., 2001), contribuindo para a reciclagem de nutrientes. Neste caso, estes insetos podem agir como conversores da qualidade do solo, tornando-o mais fértil. Quando estes processos naturais desaparecem, os custos ambientais e socioeconômicos podem ser significativos (ALTIERI et al., 2003). Levando em consideração as 
informações acima citadas, Luz et al., (2013) em levantamento da fauna de artrópodes edáficos realizado com armadilhas de bandeja d'água, observaram que as formigas foram as mais abundantes representantes da ordem Hymenoptera em áreas sob interferência antrópica e áreas preservadas, diminuindo significativamente o número de insetos capturados em áreas preservadas.

Os Diptera das famílias Drosophilidae e Muscidae apresentaram abundâncias de 316 e 213 insetos, respectivamente. Segundo Nere et al. (2011), os drosofilídeos foram mais abundantes devido ao seu hábito alimentar por frutas em processo de fermentação caídos das fruteiras na área agrícola. Já as moscas domésticas ocorreram em maior número devido a proximidade das residências rurais e criações de animais domésticos. Em avaliação da fauna de artrópodes edáficos em áreas de pastagem com uso de armadilhas d'água, a família Sarcophagidae apresentou-se mais representativa em termos de indivíduos capturados (LUZ et al., 2013), o que demostra que em áreas agrícolas de maior diversidade de cultivo e atividades agropecuárias atraem indivíduos com uma maior amplitude com relação aos hábitos alimentares.

Esse ecossistema apresentou menor diversidade em número de famílias mais abundantes do que o anterior devido a maior ação antrópica nas implantações das culturas agrícolas, construção de residências e criações de animais domésticos quando comparado com o fragmento de floresta.

No Arajara Park foram coletados 1.128 insetos e somente as formigas (Formicidae), pernilongos (Tipulidae), cigarrinhas (Cicadellidae) e as mosquinhas lambe olhos (Chloropidae) foram mais abundantes com 313, 121, 119 e 100 insetos, respectivamente. As formigas tem alto potencial de serem empregadas como modelo em estudos de biodiversidade, além de algumas espécies serem indicadoras do nível de antropização do ambiente, pois habitats complexos como áreas de lazer criam oportunidades de instalação e sobrevivência de um número maior de espécies, em virtude da capacidade de suporte do meio (ANDOW, 1991). Já os tipulídeos necessitam de habitats aquáticos ou semi-aquáticos durante a fase larval, sendo estes, córregos, nascentes e bromélias entre outros, ambientes estes encontrados no Arajara Park. A presença das cigarrinhas deve-se também a diversidade de plantas no local, pois são sugadores de seiva e a presença de cloropídeos ocorreu em virtude de existir no balneário alguns animais silvestres, mas é muito incômoda para o homem, causando desconforto em atividades recreativas e de lazer, trazendo prejuízos para o turismo.

\subsection{Insetos coletados na estação chuvosa}

Nessa estação capturou-se um total de 3.506 insetos, número este três vezes menor do que foi coletado na estação seca (Tabela 1), sendo que na área agrícola capturou-se um maior número de insetos (1.460). Nesse ecossistema, os Diptera das famílias Chloropidae, Dolychopodidae e Drosophilidae foram mais abundantes com 262, 140 e 121 insetos, respectivamente. A dominância destas famílias também foi observada por Moura et al., (2012) em trabalho realizado em área agrícola com o uso de bandejas amarelas.

Os Chloropidae atingiram este número elevado devido à existência de animais domésticos neste ecossistema, assim como, os Dolychopodidae, devido à presença de pragas agrícolas, já que nessa família existem espécies predadoras (TRIPLEHORN; JONNSON, 2011). Nessa área os Drosophilidae desempenham papel fundamental nas cadeias saprofíticas, pois suas larvas 
alimentam-se de organismos fermentadores em frutos cultivados (YEATES; WIEGMANN, 2005).

Os Hymenoptera das famílias Formicidae e Ichneumonidae apresentaram abundâncias de 168 e 150 insetos, respectivamente. As formigas podem desempenhar papel importante na dinâmica da produção de grandes culturas e frutífera e no êxito do sistema de produção agrícola (HANEY, 1988). Na maioria dos casos, existe um complexo de espécies de formigas presente no pomar, sendo algumas benéficas, outras indiferentes e uma ou duas são consideradas pragas importantes. Já os parasitoides Ichneumonidae desempenham um papel importante na área agrícola para o controle de pragas agrícolas, principalmente nessa estação que ocorre um grande número delas devido a uma maior disponibilidade de alimentos e condições favoráveis.

Os Hemiptera da família Cicadellidae apresentaram abundância de 155 insetos, por serem pragas agrícolas, principalmente de feijoeiro, cultura esta encontrada nesse ecossistema na estação chuvosa. Em vegetação de mata úmida e na estação chuvosa da Floresta Nacional do Araripe, Azevedo et al. (2011) observaram um número semelhante desses insetos de 87 indivíduos.

Em seguida, a mata úmida apresentou o maior número de insetos (789), observando-se maior abundância das cigarrinhas e formigas, com 160 e 136 insetos, respectivamente. As cigarrinhas por serem fitófagas são reguladoras das populações de plantas devido a grande diversidade principalmente na estação chuvosa e as formigas são de extrema importância para este ecossistema, pois atuam degradando matéria orgânica como a serrapilheira e participando da ciclagem de nutrientes no solo por ser um ambiente equilibrado (LEVY; BYRNE, 1993).

No fragmento com 648 insetos capturados, observou-se apenas maior abundância das cigarrinhas com 234 insetos, demonstrando baixa diversidade deles em abundância para esta estação. Como já comentado anteriormente, esses insetos preferem habitat constituído por vegetações baixas (VAZ et al., 2009) e, provavelmente, pelo fato da vegetação do fragmento apresentar plantas mais baixas, essa condição propiciou maior atividade de voo dessas cigarrinhas entre as plantas e maior captura nas bandejas.

No Arajara Park com 609 insetos capturados, percebeu-se maior abundância para as formigas e vaquinhas. A presença de árvores e a proximidade da mata úmida influenciam diretamente na presença das formigas, já que as formigas arborícolas utilizam estruturas desenvolvidas pelas plantas como dilatações de folhas e ramos, cavidades em fustes para construírem seus ninhos e implantar suas colônias, pois na estação chuvosa existem muitas árvores mortas nesse ambiente. Já as vaquinhas são insetos fitófagos, altamente relacionados à sua planta hospedeira e muitas espécies são pragas de plantas cultivadas, causando injúrias nas flores e área foliar (adultos) ou danificando as raízes e o caule (larvas) (TRIPLEHORN; JONNSON, 2011).

\section{CONCLUSÕES}

A estação seca da Área de Proteção Ambiental da Chapada do Araripe favorece a maior ocorrência de famílias abundantes que a estação chuvosa devido à alteração da disponibilidade de condições e recursos, propiciando a coexistência de espécies generalistas.

A ordem Coleoptera foi a mais rica em famílias nas duas estações do ano tendo como papel ecológico os Crysomelidae como fitófagas e Nitidulidae como decompositoras de frutas. 
Os Diptera são numerosos na estação chuvosa atuando no sistema ecológico as moscas frugívoras (Drosophilidae), decompositoras de carcaças de animais (Muscidae) e predadoras (Dolychopodidae), enquanto que os Tipulidaee Chloropidae predominam na estação seca da mata úmida.

Os Hemiptera da família Cicadellidae (cigarrinhas) ocorrem abundantemente na área agrícola e no fragmento de floresta por serem ecossistemas antropizados para a implantação de culturas agrícolas e pastagens.

Na estação seca os Hymenoptera da família Formicidae (formigas) predominam e ocorrem em maior abundância em todos os ecossistemas por serem cosmopolitas e bem adaptados, além de apresentarem hábitos alimentares generalistas.

A bandeja d'água amarela é eficiente na captura de insetos edáficos, demonstrando nas avaliações das coletas uma considerável diversidade de famílias em todos os ecossistemas avaliados assim como nas diferentes estações (seca e chuvosa), permitindo assim a ampliação de conhecimentos sobre a composição da entomofauna da Área de Proteção Ambiental do Araripe e fornecendo uma base de informações consolidada para estudos futuros.

\section{REFERÊNCIAS BIBLIOGRÁFICAS}

1. ALENCAR, A.; NEPSTAD, N.; MCGRATH, D.; MOUTINHO, P.; PACHECO, P.; DIAZ, M.D C.V.; FILHO, B. S. Desmatamento na Amazônia: indo além da emergência crônica. Instituto de Pesquisa Ambiental da Amazônia (Ipam), Manaus, p. 89,2004.

2. ALONSO, L.E. Ants as indicators of diversity. In: AGOSTI, D.; MAJER, J.D.; ALONSO, L. E.; SCHULTZ, T.R. (Eds). Ants: standard methods for measuring and monitoring biodiversity. Washington: Smithsonian Institution, p. 80-88, 2000,

3. ALTIERI, M.A.; SILVA, E.N.; NICHOLLS, C. I. O papel da biodiversidade no manejo de pragas. São Paulo: Holos, 215p. 2003.

4. ANDOW, D. A. Vegetacional diversity and arthropod population responses. Annual Review of Entomology, v.36, p.561-586, 1991.

5. ARAUJO, L.H.A. Novos sistemas de tomada de decisão a serem usados no manejo do pulgão Aphis gossypii (GLOVER, 1877) (HEMIPTERA: APHIDIDADE) na cultura do algodoeiro. 103f. Tese (Doutorado). Universidade Federal da Paraíba, Areia, 2009.

6. ARAÚJO, M.V.JR. Efeito da fragmentação florestal nas taxas de herbivoria da formiga cortadeira Atta laevigata. Dissertação de Mestrado, 86f. Universidade Federal de Pernambuco, Recife, 2004.

7. ARAÚJO, C.C.; NOMELINI, Q.S.S.; PEREIRA, J.M.; LIPORACCI, H.S.N.; KATAGUIRI,V.S. Comparação da abundância de invertebrados de solo por meio da estimação intervalar encontrados em diferentes ambientes na cidade de Ituiutaba - MG. Bioscience Journal, v.26, n. 5, p.817-823, 2010.

8. ATHIÉ, I.; PAULA, D. C. Insetos de grãos armazenados: aspectos biológicos e identificação. 2a ed.,SãoPaulo: Livraria Varela, 2002, 244p.

9. AZEVEDO, F.R.; MOURA, M.A.R.; ARRAIS, M.S.B.; NERE, D.R. Composição da entomofauna da Floresta Nacional do Araripe em diferentes vegetações e estações do ano. Revista Ceres, 
v.58, n.6, p. 740-748, 2011.

10. BEGON, M.; TOWNSEND, C. R.; HARPER, J. L. Ecologia de indivíduos a ecossistemas. 4aed., PortoAlegre: Artmed, 2008, 752p.

11. BUZZI, Z.J. Entomologia didática.4a ed. Curitiba: Editora UFPR, 2008, 348p.

12. CAMPOS, W.G.; PEREIRA, D.B.S.; SCHOEREDER, J.H. Comparison of the efficiency of flightinterception trap models for sampling Hymenoptera and other. Anais Sociedade Entomológica Brasileira, v.29, p.381-389, 2000.

13. CARVALHO, C.J.B. Dolichophaonia, gen. (Diptera, Muscidae, Phaoniinae): descrições, novas combinações, sinonímias e chave para as espécies. Revista Brasileira de Entomologia, v.37, p.19-34, 1993.

14. CHUNG, A.Y.C, EGGLETON, P. SPEIGHT, M.R; HAMMOND, P.M; CHEY, V.K. The diversity of beetle assemblages in different habitat types in Sabah, Malaysia. Bulletin of Entomological Research, v.90, p.475-496, 2000.

15. COELHO, L.B.N; SILVA E.R. Flutuação populacional de Agallia incongrua Oman,1938 (Hemiptera: Cicadellidae) em Viçosa, Minas Gerais, Brasil. Biota Neotrópica, n.3,p.1-8, 2003.

16. DAVIDSON, D.W.; COOK, S. C.; SNELLING, R. R.; CHUA T. H. Explaining the Abundance of Ants in Lowland Tropical Rainforest Canopies. Science, v.300, p.969-972, 2003.

17. DEL-CLARO, K.; SANTOS, J. C.; DURÃES, A. S. J. Etograma da formiga arborícola Cephalotes pusillus (Klug, 1824) (Formicidae: Mirmicinae). Revista de Etologia, v.4, n.1, p. 31-40, 2002.

18. FARJI-BRENER, A.G.; ILLES, A.E.Do leaf-cutting ant nests make "bottom-up" gaps in neotropical rain forest? A critical review of the evidence. Ecology Letters, n.3, p.219-227, 2000.

19. GALLO, D.; NAKANO, O.; NETO, S. S.; CARVALHO, R. P. L.; BAPTISTA, G. C.; de; BERTI FILHO, E.; PARRA, J. R. P.; ZUCCHI, R. A.; ALVES, S. B.; VENDRAMIM, J. D.; MARCHINI, L. C.;LOPES, J. R. S.; OMOTO, C. Entomologia Agrícola. Piracicaba: FEALQ, 2002, 920p.

20. GUARIENTO. H. F.; Levantamento Entomológico na Fazenda Prata, São João da Boa Vista, SP. Monografia apresentada como parte da disciplina de estagio supervisionado do curso de ciências biológicas do Centro Universitário da Fundação de Ensino Octavio Bastos São João da Boa Vista, SP, 2005.

21. HANEY, P.B .1988. Identification, ecology and control of the ants in citrus: a world survey. In: Goren R, Mendel K (eds) Proceedings of the sixth International Citrus Congress, Tel Aviv, Israel, pp 1227-1251.

22. HÖFER, H.; HANAGART, W.; GARCIA, M.; MARTIUS, C.; FRANKLIN, E.; RÖMBKE, J.; BECK, L. Struture and function of soll fauna communities in Amazonian anthropogenic and natural ecosystems. Euro Journal of Soil Biology, v.37, p.229-235, 2001.

23. ISERHARD, C.A.; KAMINSKI, T.A.; TEIXEIRA, E.C.; MARCHIORI, M.O.; ROMANOWSKI, H.P. Levantamento preliminar da entomofauna noturna ocorrente no município de Três Coroas, RS. In: CONGRESSO DE ECOLOGIA DO BRASIL,5. Resumos... Porto Alegre - RS, 2001.

24. YEATS, D.K,; WIEGMANN, B.H. Phylogeny and Evolution of Dipter: Recent insights and new perpectivesIn: YEATS D.K.; WIEGMANN, B.M. (Eds).The evolutionary biology of flies.Columbia University Press, New York,p. 14-44, 2005. 
25. KAGEYAMA, P.; LEPSCH-CUNHA, N. M. 2001. Singularidade da biodiversidade nos trópicos. In: GARAY, I. \& DIAS, B. (eds.). Conservação da biodiversidade nos trópicos, Ed. Vozes, Petrópolis, p. 199-214.

26. LASALLE, J.; I. D. GAULD. 1993. Hymenoptera and biodiversity.C. A. B. International. 348p. LAURANCE, W. L.; ALBERNAZ. A. K. M.; FEARNSIDE, P. M.; VASCONCELOS, H.; FERREIRA, L. V. "Deforestation in Amazonia". Science, v.304, p.1109-1111, 2004.

27. LEMCKERT, F. Parasitism of the common eastern froglet Criniasigniferaby flies of the genus Batroachomyia (Diptera: Chloropidae): Parasitism rates and influence on frog condition. Australian Zoology. v.31, p.492-495, 2000.

28. LEVEY, J.; M.M. BYRNE. Complex Ant-Plant Interactions: Rain Forest Ants as Secondary Dispersers and Pos-Dispersal Seed Predators. Ecology, v.74, p.1802-1812, 1993.

29. LEWINSOHN, T.M.; FREITAS, A.V.L.; PRADO, P.I. Conservation of terrestrial invertebrates and their habitats in Brazil. Conservation Biology, v.19, p.640-645, 2005.

30. LONGINO, J. T; COLWELL, R. K. Biodiversity assessment using structured inventory: capturing the ant fauna of a Tropical Rain Forest. Ecological Applications, v.7, n.4, p.1263-77, 1997.

31. LOPES, B.G.C. Levantamento da entomofauna bioindicadora da qualidade ambiental em diferentes áreas do alto Jequitinhonha- Minas Gerais. Monografia de Graduação, 47f, Escola Agrotécnica Federal de Inconfidentes, Inconfidentes, 2008.

32. LUZ, R.A.; FONTES, L.S.; CARDOSO, S.R.S.; LIMA, E.F.B. Diversity of the Arthropod edaphic fauna in preserved and managed with pasture areas in Teresina-Piauí-Brazil. Brazil Journal Biology, v.73, n. 3, p.483-489, 2013.

33. MELO, L.A.S.; MOREIRA, A.N.; SILVA, F.A.N. Armadilha para monitoramento de insetos. Embrapa Meio Ambiente: Jaguariúna-SP, 2001, 4p.

34. MONSEVIÈIUS, V. Comparison of three methods of sampling wild bees (Hymenoptera, Apoidea) in Èepkeliai Nature Reserve (South Lithuania). Ekologija, v. 4, p. 32-39, 2004.

35. MOURA, E. S.; AZEVEDO, F. R.; SANTOS, C. A. M.; NERE, D. R.; AZEVEDO,R. Bandeja d'água de cor amarela na captura de insetos em ecossistemas da Apa Araripe. In: ENCONTRO UNIVERSITÁRIO DA UFC NO CARIRI, 4, Resumos...Juazeiro do Norte-CE, 2012.

36. NASCIMENTO, R.P.; MORINI, M.S.C.; BRANDÃO, C.R.F. Mirmecofauna do Parque natural municipal da Serra do Itapety. Zona de uso intensivo. In: ENCONTRO DE MIRMECOLOGIA, 15, 2001. Londrina. Resumos... Londrina: IAPAR, 2001. p.339-341.

37. NERE, D. R.; MARIANO, C. A.; MOURA, E. S.; AZEVEDO, F. R. Captura de insetos de voo baixo em bandejas amarelas em Ecossistemas do Cariri Cearense. In: 3o ENCONTRO UNIVERSITÁRIO DA UFC NO CARIRI, 3, Resumos... Juazeiro do Norte-CE, 2011.

38. PEREIRA, J.A., BENTO, A., CABANAS, J.E., TORRES, L.M., HERZ, A., HASSAN, S.A. Ants as predators of the egg parasitoid Trichogramma cacoeciae (Hymenoptera: Trichogrammatidae) applied for biological control of the olive moth, Prays oleae (Lepidoptera: Plutellidae) in Portugal. Biocontrol Science Technology, v.14, p.653-664. 2004.

39. SANTOS, M. S.; LOUZADA, J. N. C.; DIAS, N.; ZANETTI, R.; DELABIE, J. H. C.; NASCIMENTO, I. C. Riqueza de formigas (Hymenoptera, Formicidae) da serapilheira em fragmentos de floresta semidecídua da Mata Atlântica na região do Alto do Rio Grande, MG, Brasil. Iheringia, Série Zoologia, v.96, n.1, p.95-101, 2006. 
40. SANTOS S.A.P.; PEREIRA, J.A.; TORRES, L.M.; NOGUEIRA, A.J.A. Evaluation of the effects, on canopy arthropods, of two agricultural management systems to control pests in olive groves from north-east of Portugal. Chemosphere, v.67, p.131-139, 2006.

41. SILVA, R. A. Levantamento de insetos no cerrado amapaense. In: REUNIÃO ANUAL DO INSTITUTO BILÓGICO, São Paulo. O biológico, 2006.

42. SCHOEREDER, J. H.; SOBRINHO, T. G.; RIBAS, C. R.; CAMPOS, R. B. F. Colonization and extinction of ant communities in a fragmented landscape. Austral Ecology, v.29, n.391-398, 2004.

43. SCHORN, L. A. Fatores que afetam a produção de sementes (Curso de manejo e conservação de sementes de espécies arbóreas da Mata Atlântica - Região Sul), 1. Blumenau. 47p., 2000.

44. TEIXEIRA, F.M. Capture techniques for Hymenoptera (Insecta). Vértices, v.14, n.1, p. 169198, 2012.

45. THOMAZINI, M.J., THOMAZINI, A.P.B.W. A fragmentação florestal e a diversidade de insetos nas florestas tropicais úmidas. Rio Branco: Embrapa Acre, 21p, 2000. Embrapa Acre. (Documentos, 57).

46. THOMAZINI, M.J., THOMAZINI, A.P.B.W. Levantamento de insetos e análise entomofaunística em floresta, capoeira e pastagem no sudeste acreano. Rio Branco: Embrapa Acre, 41p.,2002. (Boletim de Pesquisa e Desenvolvimento, 35).

47. TOGNON, R.; FERRI, D.; AZEVEDO FILHO, W. S. Cigarrrinhas (Cicadellidae: Cicadellinae, Proconiini) potenciais vetores de Xylella fastidiosa em pomares de Citrus deliciosa (Rutaceae) no Rio Grande do Sul, Brasil. In: X Salão de Iniciação Científica PUCRS. São Paulo, 2009.

48. TRIPLEHORN, C.A; JONNSON, N.F. Estudo dos insetos. São Paulo: Cengage Learning. 2011, $809 p$.

49. VAZ, V.V.A.; DUMMEL, K.; NUNES, M.R.; GANTES, M.L.; OLIVEIRA, E.A.; CARRASCO, D.S.; ZARDO, C.M.L. Comparação de Cicadellidae (Hemiptera; Auchenorrhyncha) em duas ilhas com diferentes composições florísticas, situadas no estuário da laguna lagoa dos patos, RS, Brasil. In: CONGRESSO DE ECOLOGIA DO BRASIL, 9, São Lourenço - Minas Gerais, Anais, CDROM, 2009.

50. VEIGA-FERREIRA, S.; MAYHÉ-NUNES A. J.; QUEIROZ J. M. Formigas de serapilheira na reserva biológica do Tinguá, Estado do Rio de Janeiro, Brasil (Hymenoptera: Formicidae). Revista Universidade Rural, Série Ciência e Vida. v.25, n.1, p.49-54, 2005.

51. WINK, C., GUEDES, J.V.C. FAGUNDES, C.K.; ROVEDDER, A.P. Insetos edáficos como indicadores da qualidade ambiental. Revista de Ciências Agroveterinárias, n.4, p.60-71, 2005.

52. WIRTH, R.; BEYSCHLAG, W.; HERZ, H.; RYEL, R.J.; HÖLLDOBLER, B. Herbivory of leaf-cutter ants: a case study of Atta columbica in the tropical rainforest of Panama. Ecological Studies, v.164, p.1-233, 2003. 